\title{
Libéralisation du service public et action publique locale. Le département dans la recomposition du système de distribution électrique français.
}

François-Mathieu POUPEAU

Avec l'accélération de la mondialisation et du phénomène de métropolisation, avec l'émergence de nouveaux modèles productifs ${ }^{1}$, le rapport de l'économie aux territoires s'est profondément modifié au cours de la dernière décennie. Manifeste dans les activités industrielles "classiques", cette transformation affecte également les services publics organisés en réseaux et placés, en France, sous la tutelle de l'État que sont les télécommunications, la distribution d'électricité et de gaz ou encore le transport ferroviaire. Après avoir étroitement épousé l'architecture institutionnelle nationale en accompagnant le développement de l'État-Providence, ces secteurs sont aujourd'hui en voie de recomposition territoriale. Soucieux de maintenir leur compétitivité dans un environnement de plus en plus concurrentiel, les opérateurs historiques reconsidèrent leur implantation. Ils s'appuient sur des innovations comme les progrès des technologies de l'information et de la communication pour réduire leurs effectifs et redéfinir leurs structures. Ils participent au vaste mouvement de concentration économique pour se déployer à l'étranger et atteindre une dimension mondiale. Sur un autre plan, celui de la régulation publique ${ }^{2}$, les apports récents de la science économique renouvellent les formes d'organisation et d'encadrement du marché. La diffusion de la théorie des "marchés contestables" (Baumol et al., 1982) opère une véritable révolution car elle permet de penser de façon distincte deux éléments longtemps perçus comme indissociables, à savoir le transport physique du bien voix, électricité, gaz, personnes, marchandises... - et sa commercialisation services associés à la fourniture (Curien, 1992). Alors que l'activité de transport

\footnotetext{
* chercheur associé au Centre de Sociologie des Organisations, 19 rue Amélie, 75007 Paris.

1 ce qui serait le passage d'une organisation de type fordiste à des formes de coordination plus souples, plus flexibles, dans lesquelles la coopération, la relation avec de multiples partenaires sont devenues essentielles (Veltz, 1996).

2 nous entendons ici le terme de régulation dans son acception anglo-saxonne, c'est-à-dire comme l'ensemble des principes et mécanismes par lesquels la puissance publique organise et contrôle un secteur économique donné.
} 
reste ouverte à un nombre restreint d'entreprises ${ }^{3}$, la commercialisation du bien peut être, quant à elle, sujette à une mise en concurrence. Cette dissociation a des conséquences sur l'organisation des secteurs libéralisés. Elle rend plus complexe leur fonctionnement et leur rapport aux territoires, dans la mesure où les contraintes industrielles et financières traditionnelles doivent composer avec d'autres logiques ${ }^{4}$.

Ces transformations alimentent bon nombre de débats sur l'action publique locale. Une première série de travaux porte sur le thème de la régulation du service public (Flux, 1998). Elle s'interroge sur la capacité des collectivités locales à peser sur les décisions des opérateurs et à les associer à la gestion de problèmes économiques, sociaux, environnementaux de plus en plus “territorialisés". Une seconde série de travaux, plus ancienne dans son questionnement, se décentre de cette problématique pour s'intéresser à l'impact des transformations sur le mode de gouvernement local (Lorrain, 1991; Lorrain, 1993). La question est de savoir si le poids croissant des grandes entreprises dans les villes marque une rupture radicale dans l'analyse de l'action publique locale et si elle participe ou non de ce que certains appellent la "gouvernance urbaine" (Le Galès, 1995; Lorrain, 1998 ; Jouve et Lefèvre, 1999).

Au-delà des différences qui les séparent, ces travaux ont en commun de porter un regard privilégié sur l'étude du monde urbain. Il est vrai que les grandes agglomérations sont depuis plusieurs années au coeur des mutations de la société française, que ce soit sur le plan politique, économique ou social. L'urbanisation et la métropolisation apparaissent comme des phénomènes durables, dont les conséquences sont encore loin d'être mesurées. On peut toutefois se demander, dans un contexte où l'État, tutelle historique du service public national, redéfinit son rôle, quelle va être la place de collectivités comme la région ou le département. Va-t-on vers une montée en puissance de la région, aux côtés des villes, dans l'encadrement du service public ? Ou assiste-t-on à un positionnement du département? Afin d'apporter un premier éclairage à ce qui nous paraît être un

\footnotetext{
${ }^{3}$ situation qui va du monopole dans le cas du transport à très haute tension d'électricité à une forme d'oligopole dans le cas du téléphone. En effet, la multiplication sauvage des lignes électriques et téléphoniques, des tuyaux de gaz... induirait des nuisances et des surcoûts.

4 comme une plus forte pression de la sphère financière (actionnariat à la recherche d'une rentabilité à court terme, spéculation boursière...). Un bon exemple en est le fonctionnement du marché britannique de l'électricité (Henry, 1997).
} 
axe de recherche stimulant, nous prendrons l'exemple du système de distribution électrique français, qui est en pleine recomposition avec la perspective d'une ouverture du marché à la concurrence ${ }^{5}$. Nous montrerons le rôle essentiel joué par le système départemental dans le redéploiement d'EDF. Bien loin de subir le processus de libéralisation et un peu paradoxalement dans un contexte de mondialisation, le département, qui est souvent décrié pour ce qui serait son archaïsme, apparaît dans toute sa vitalité et sait tirer profit des remises en question actuelles pour se repositionner sur la scène locale.

Pour ce faire, après avoir montré que le "modèle EDF" (Wieviorka et Trinh, 1989) a toujours été soumis à des interactions avec le monde local, notamment rural, nous combinerons deux approches pour appréhender les changements récents. La première s'intéresse au cadre de la régulation publique. Elle met en évidence une " reterritorialisation" $\mathrm{d}$ 'EDF, qui opère un rapprochement sensible avec ses partenaires locaux dans les années 1990. La seconde s'efforce de rentrer un peu plus dans le fonctionnement quotidien de l'entreprise. Analysant la mise en oeuvre d'un nouveau modèle d'organisation de la distribution, elle montre une certaine " déterritorialisation" d'EDF, du point de vue de son implantation locale. C'est dans l'articulation de ces deux dynamiques, apparemment contradictoires, qu'il faut lire le renforcement du système départemental.

\section{LA RÉGULATION TERRITORIALE DU “MODÈLE EDF"}

A la veille du processus de libéralisation, les unités d'EDF-GDF6 sont l'objet de nombreuses relations avec les collectivités territoriales. Même si elle demeure discrète dans un système qui est très contrôlé par l'Etat, moteur de l'économie administrée depuis l'après-guerre, l'influence locale s'exerce de deux manières. De façon formelle, dans le cadre du régime de l'électrification rurale, qui a été maintenu au moment de la nationalisation. De façon informelle, à travers les

\footnotetext{
${ }^{5}$ nous privilégierons l'activité de distribution car elle se situe à la charnière entre les logiques industrielle et territoriale du secteur électrique. Les dimensions " transport" et "production" ne seront abordées que lorsqu'elles pourront enrichir nos propos. En outre, centrant notre analyse sur $\mathrm{EDF}$, nous ne nous intéresserons pas au cas des opérateurs locaux $(5 \%$ de la distribution d'électricité en France), dont la problématique est trop spécifique pour être correctement traitée dans cet article.

${ }^{6}$ la Direction de la Distribution (EDF-GDF) est commune à EDF et à GDF, ainsi que la Direction du Personnel et des Relations Sociales (DPRS) et la Direction des Affaires Générales (DAG).
} 
multiples interactions qui existent au quotidien entre les agents d'EDF-GDF et les élus locaux. Non sans rappeler certains traits du "modèle d'administration territoriale républicaine" (Grémion, 1976), ces ajustements relativisent l'image jacobine qui reste souvent attachée à l'entreprise publique.

\subsection{Un ajustement politique : le maintien de l'électrification rurale}

L'instauration d'EDF ne se fait pas dans un vide institutionnel. Au contraire, c'est à partir d'un riche tissu local, conforté par la loi du 8 avril 1946 nationalisant les anciennes sociétés privées, que s'opère la constitution du "modèle EDF". En effet, au sortir de la guerre, les collectivités locales, qui avaient joué un rôle prépondérant dans le développement de l'électricité, voient leurs compétences réaffirmées. Sur le plan juridique, elles demeurent les autorités concédantes de la distribution7. Celles qui avaient instauré des régies (ou équivalents) - qui représentent alors environ $5 \%$ de la consommation totale d'électricité - sont autorisées à les conserver. Pour donner corps à ces prérogatives, le législateur prévoit de décentraliser l'activité de distribution au sein d'établissements publics locaux, qui doivent servir de contrepoids face à EDF, qui détient un monopole en matière de transport et un quasi-monopole en matière de production.

Rapidement, l'exercice de ces fonctions devient virtuel dans un contexte où l'État maîtrise les ressources essentielles que sont la politique d'investissement et de tarification de l'entreprise publique. Soutenue par le ministère des Finances, la CGT mais aussi EDF, qui voient d'un mauvais oeil l'existence d'un contre-pouvoir local, la centralisation finit par s'imposer ${ }^{8}$. Privées des deux principaux attributs du régime de la concession - la tarification, les investissements -, les communes sont reléguées à un rôle secondaire. Mais ce dessaisissement progressif n'est pas sans contrepartie. Face à un lobby local très organisé autour de la Fédération Nationale des Collectivités Concédantes et Régies (FNCCR), qui a été créée en 1933 pour représenter les intérêts des communes sur le plan national, l'État et EDF doivent rechercher certains compromis. De cette volonté d'apaisement procède le maintien du régime de l'électrification rurale, créé durant l'entre-deux-guerres pour

\footnotetext{
7 s'agissant des usagers pour lesquels la tension délivrée est inférieure à 63.000 volts (loi du 15 juin 1906). Au-delà, l'État se substitue aux communes et groupements de communes.

${ }^{8}$ pour une analyse détaillée de ce processus, cf. Bonaïti, 1997 ; Poupeau, 1999.
} 
hâter la modernisation des campagnes. Alors qu'il aurait pu être placé sous la seule responsabilité d'EDF, ce dispositif, qui est étroitement contrôlé par les élus, continue de subsister. Au fil des années, malgré l'achèvement de l'électrification, il se mue en "chasse gardée" du monde rural et ne cesse de drainer des sommes substantielles ${ }^{9}$.

Dans ce domaine, l'avènement d'EDF ne modifie guère la répartition des compétences qui existait antérieurement. Celle-ci fait intervenir quatre principaux protagonistes. En tant que maîtres d'ouvrage, les élus locaux conservent leur pouvoir de décision en matière d'équipement des réseaux électriques de basse et moyenne tension pour les communes de moins de 2000 habitants. Ils sont organisés en syndicats primaires. Deux ou trois assemblées générales, qui rassemblent les délégués des communes, se déroulent chaque année pour définir la liste des travaux à effectuer. Une structure départementale, fédération de ces syndicats primaires, complète parfois le dispositif, dans la mesure où les crédits sont affectés par département, sous l'autorité du préfet, le dispensateur des crédits publics. Ces structures intercommunales disposent rarement de leur propre équipe d'ingénieurs et de techniciens leur permettant de remplir une fonction de maîtrise d'oeuvre. Dans la majorité des cas, celle-ci est assurée, contre rémunération, par les services extérieurs de l'État - Ponts-et-Chaussées et Génie Rural -, en lien avec les Services Techniques Électricité (STE) d'EDF. Chargés de concrétiser les décisions politiques, ces intervenants montent les dossiers techniques. Ils les confrontent aux contraintes des exploitants, agents d'EDF qui sont le dernier maillon de la chaîne de décision. Responsables du fonctionnement du réseau, ces derniers ont leur mot à dire car certaines options peuvent avoir une incidence sur la qualité de la fourniture d'électricité.

C'est autour de ces quatre principaux acteurs - les élus, le préfet, les techniciens, les exploitants - que se définit la politique départementale d'électrification rurale. $\mathrm{Au}$ sortir de la guerre, les choix d'investissements sont fortement politisés, dans la mesure où la capacité d'un élu à hâter l'électrification d'une commune renforce sa légitimité et son assise locales. Les assemblées générales sont souvent mouvementées et les tractations fréquentes. Aussi retrouve-t-on la plupart des

9 de l'ordre de 2,85 milliards de francs (francs constants de 1990) pour le deuxième Plan (1953-
1956) (Morsel, 1996). Aujourd'hui encore, l'ensemble des crédits avoisine les 4 milliards de francs. 
caciques locaux au sein des instances délibérantes. Avec la fin de l'électrification en surface, les décisions perdent, certes, de leur visibilité politique. Lorsqu'il s'agit non plus d'étendre mais de renforcer un réseau sous-dimensionné, les contraintes des ingénieurs et exploitants d'EDF se font plus prégnantes car les choix réalisés sur les basse et moyenne tensions doivent être de plus en plus compatibles avec la planification prévue par l'entreprise. L'espace de négociation se trouve ainsi reconfiguré au profit d'une logique plus technique. Il n'en demeure pas moins que chaque phase de développement des réseaux est porteuse d'enjeux nouveaux. Le renforcement des lignes coïncide avec une période, les Trente Glorieuses, où les ménages s'équipent massivement en appareils électriques. La maîtrise de l'allocation des ressources reste donc stratégique car elle permet de réduire des délais de travaux parfois très longs. Elle le demeure lorsqu'EDF entame une démarche d'amélioration de la qualité du courant ou lorsque, plus récemment, l'enfouissement des lignes est recherché pour réduire les nuisances visuelles dans les centres-bourgs. C'est donc avec une logique politique que les centres de distribution doivent constamment composer pour construire leurs réseaux en zone rurale et assumer leur mission de service public.

\subsection{Un ajustement organisationnel : une conception pragmatique du service public}

Cette première forme de régulation ${ }^{10}$ doit être complétée par d'autres modes d'influence moins explicites car s'exerçant de façon informelle entre les centres EDF-GDF et les élus locaux. Pour bien en comprendre la nature, il convient de rentrer un peu plus dans l'organisation interne de la Direction de la Distribution. $\mathrm{Au}$ lendemain de la nationalisation, c'est un schéma assez traditionnel, calqué sur les Ponts-et-Chaussées, qui s'instaure. Il repose sur une organisation pyramidale, constituée d'une centaine de centres, qui correspondent plus ou moins aux départements. Au milieu des années 1950, ces structures sont complétées par des Directions Régionales, dont la fonction essentielle est d'être des intermédiaires de gestion entre les centres et Paris.

10 ici et plus loin, ce terme sera utilisé non plus au sens donné en introduction mais comme l'ensemble des mécanismes par lesquels un système social se maintient et se reproduit (Crozier et Friedberg, 1977). 
Les centres sont organisés suivant deux principes : par filière verticale (technique, clientèle, commerciale...) et par unité territoriale (subdivisions, districts). Les filières ont pour vocation d'assurer une cohérence nationale en uniformisant les pratiques internes. Ainsi, les conditions de raccordement d'un usager, les pénalités à verser en cas d'impayé ou la facturation de certaines prestations, qui dépendaient auparavant des cahiers des charges des concessions locales, sont définies par les services parisiens de la Distribution. Les unités territoriales, qui regroupent des agents de plusieurs filières, sont conçues, quant à elles, comme une reproduction des centres à une plus petite échelle. Un centre compte en général de deux à quatre subdivisions, qui, elles-mêmes, regroupent en moyenne trois districts. Ces unités créent un maillage fin, sur lequel l'entreprise peut s'appuyer pour moderniser les réseaux. La répartition des responsabilités entre structures territoriales répond à une division du travail précise : aux centres sont déléguées la gestion des plus gros travaux d'investissement et d'exploitation (lignes à haute voire moyenne tension) et la gestion des contrats les plus importants (tarifs verts, voire jaunes ${ }^{11}$ ), aux districts la gestion des investissements quotidiens et des contrats bleus. Les tâches confiées aux subdivisions varient suivant les périodes et les centres. Dans ce mode d'organisation " matriciel " et dans une entreprise qui reste fondée sur une logique de production ${ }^{12}$, le poids des filières est structurant. Il contribue à cloisonner les services au sein d'une même unité territoriale, chaque agent se référant avant tout aux principes définis par les services centraux.

Dans un tel modèle, que l'on peut qualifier de "bureaucratique" car davantage fondé sur une logique interne qu'externe - la satisfaction du client -, les relations à l'environnement local peuvent sembler a priori être dénuées d'enjeux. Cependant, à l'instar du système politico-administratif français (Crozier et Thoenig, 1975 ; Grémion, 1976), les centres EDF-GDF sont soumis à de multiples interactions avec le monde local, en particulier avec les élus. Les ajustements, qui portent surtout sur des questions de la vie quotidienne, reposent sur des dérogations nombreuses aux règles prescrites pour chaque filière. Obtenir qu'un administré bénéficie de délais de règlement plus longs, déplacer un poteau à titre gracieux, aménager le tracé d'une ligne qui suscite des mécontentements, différer des

\footnotetext{
11 à partir des années 1960, il existe trois grandes catégories de tarifs pour l'électricité, suivant la puissance installée : le tarif bleu correspond aux consommations les plus faibles, le tarif vert aux plus importantes (industriels le plus souvent). Le tarif jaune se situe entre les deux.

12 produire des $\mathrm{kWh}$, " produire" des réseaux électriques pour équiper le pays.
} 
chantiers pour les coordonner avec des travaux de voirie... sont autant de "services" qu'un maire peut demander à un chef de district ou de subdivision. Selon le poids politique de l'élu, la réceptivité et la capacité des agents d'EDFGDF à jouer avec les règles bureaucratiques internes, les demandes sont plus ou moins honorées. Accéder à celles-ci participe de la construction de bonnes relations qui pourront s'avérer utiles dans d'autres circonstances. En contrepartie d'un service rendu, un élu pourra en effet user de son influence personnelle pour persuader un agriculteur récalcitrant d'accepter l'installation d'un poteau dans son champ, sans qu'EDF n'ait à engager de procédure administrative lourde. Ce même élu pourra opter plus facilement pour l'installation d'un chauffage électrique dans un bâtiment communal, alors que des concurrents proposent des solutions compétitives au fioul ou au propane. Il pourra être un soutien précieux pour appuyer une demande vis-à-vis du centre pour l'établissement des budgets ou la promotion d'une carrière ${ }^{13}$. Au-delà des discours et d'une culture de service public égalitariste, impersonnelle, les agents d'EDF montrent une grande souplesse au quotidien, qui favorise l'assimilation de leur entreprise par la population. Ils incarnent une vision du service public pragmatique, fondée sur la dérogation et l'application négociée des règles nationales.

\subsection{L'emprise du monde rural sur le " modèle EDF"}

Ce mode de régulation bureaucratique ${ }^{14}$ se substitue progressivement aux relations contractuelles qui auraient dû lier EDF aux communes. Même si les villes ne sont pas dépourvues de moyens d'action ${ }^{15}$, l'emprise du monde rural est manifeste. Elle s'exerce de diverses manières, par l'électrification rurale mais aussi au quotidien, de façon informelle, car c'est moins le poids "commercial” de la commune qui compte que la capacité de ses élus à infléchir les règles internes à EDF-GDF. En revanche, l'influence de la région demeure très limitée, même lorsque sont créées,

\footnotetext{
13 on retrouve ici les mécanismes de la " régulation croisée " (Crozier et Thoenig, 1975).

14 " bureaucratie" devant être prise ici non pas comme idéal-type wéberien (qui en fait une entité totalement fermée à son environnement) mais au sens où l'entendent les sociologues des organisations, c'est-à-dire comme une structure certes fortement modelée par des logiques internes mais qui sait aussi s'adapter aux demandes toujours spécifiques de son environnement.

${ }^{15}$ quelques élus savent jouer de leur connaissance de la loi de 1946 pour renégocier le montant de certaines redevances, ce qui réintroduit une forme de différenciation vis-à-vis des autres communes.
} 
dans les années 1970, quelques agences régionales développant une politique de soutien aux économies d'énergie.

Soumis à des turbulences au moment des lois de décentralisation ${ }^{16}$, ce modèle territorial fait preuve d'une grande stabilité tant il contente les principaux protagonistes du secteur électrique. Les dirigeants d'EDF, les administrations d'État, la CGT voient d'un mauvais oeil la remise en cause de l'équilibre instauré après guerre, qui représente à leurs yeux un bon compromis. Ils sont rejoints par une forte majorité d'élus locaux, qui savent tirer parti du nouveau système. La position de la FNCCR reflète bien cet état d'esprit. Longtemps protestataire à l'égard de la centralisation, elle finit par s'accommoder de la situation car elle conserve de nombreux moyens d'influence. Elle est l'une des forces qui participent aux grandes décisions d'EDF en matière d'investissement ou de tarification ${ }^{17}$. Mais surtout, elle tire sa force de la position qu'elle occupe au sein du Conseil du Fonds d'Amortissement des Charges d'Électrification (FACE), organisme chargé de répartir les crédits de l'électrification rurale entre les départements. A défaut d'être majoritaire au Conseil d'Administration du FACE, la FNCCR sait jouer de certaines alliances pour influencer les grandes décisions, notamment sur l'octroi des crédits. Elle se trouve de ce fait à la tête d'un dispositif qui lui procure pouvoir et légitimité.

\section{D'UNE RÉGULATION BUREAUCRATIQUE A UNE RÉGULATION CONTRACTUELLE : LA RECOMPOSITION DU " MODÈLE EDF"}

La recomposition de ce modèle de régulation s'engage à la fin des années 1980 avec l'ouverture à la concurrence du marché français de l'électricité. Ce mouvement marque une rupture, dans la mesure où il remet en question deux des fondements du rapport d'EDF aux territoires : le mode d'intervention de l'État et la logique d'implantation locale de l'entreprise.

\footnotetext{
16 un temps évoquée par certains courants socialistes, la régionalisation d'EDF et de GDF est abandonnée. Seules quelques mesures en faveur des économies d'énergie sont mises en oeuvre.

17 par exemple lorsqu'il s'agit d'opter pour une péréquation des prix, de défendre les tarifs des petits consommateurs vis-à-vis des industriels ou de peser sur les arbitrages d'investissement entre production, transport et distribution.
} 
Le processus d'intégration européenne marque le passage d'une économie administrée à un cadre d'action reposant sur la concurrence. Remettant en question les fonctions traditionnelles d'un État à la fois actionnaire, tutelle technique et opérateur économique, il ouvre le jeu de la régulation et pose la question de la pérennité des compromis noués après-guerre entre État et pouvoir local.

Appelée à passer d'un statut de monopole protégé par l'État à celui d'un grand groupe de services international, EDF entame une refonte de son modèle d'entreprise. Celle-ci implique, en particulier, la révision d'une implantation conçue après guerre dans une logique "extensive " : il s'agissait d'être présent sur tout le territoire pour reconstruire, moderniser les réseaux puis diffuser les usages de l'électricité dans la société française. Désormais, dans un environnement qui tend à devenir plus concurrentiel, les gains de productivité sont prioritaires. Conjuguée au précédent processus - la recherche d'un nouveau cadre d'action publique -, une dynamique de "déterritorialisation" s'engage, qui modifie considérablement la nature des relations nouées avec les collectivités locales.

\subsection{Renforcer l'ancrage institutionnel d'EDF : la négociation de nouveaux contrats de concession}

A l'amorce du processus de libéralisation, un doute peut encore subsister quant à l'échelon institutionnel - État, collectivités locales - qui pourrait bénéficier de la nouvelle donne économique. En effet, quarante années de prédominance de l'État n'ont pas effacé les prérogatives locales en matière de distribution d'électricité. $\mathrm{Au}$ contraire, celles-ci ont été renforcées par les lois de décentralisation, qui autorisent les communes à négocier librement leur cahier des charges, sans passer par un document type national ${ }^{18}$. Même s'il a été contourné depuis 1946 , le régime de la concession continue donc d'être le fondement juridique du modèle français de service public et pourrait servir de base à une remise en cause du monopole d'EDF.

\footnotetext{
18 auparavant, les contrats de concession étaient soumis à une tutelle de l'État a priori, qui imposait de respecter un cahier des charges agréé par différents ministères.
} 
Tel n'est cependant pas le cas, dans la mesure où la Commission Européenne choisit le modèle de l'Accès des Tiers aux Réseaux (ATR) comme principe d'organisation du futur marché européen. Fondé sur la théorie des "marchés contestables " (Baumol et al., 1982), l'ATR ne reconnaît comme monopole naturel que l'activité de transport " physique" d'électricité, la production et la fourniture de services aux clients finals étant considérées comme étant du domaine concurrentiel. Ne s'attaquant pas directement aux systèmes nationaux de distribution, l'ATR instaure une mise en concurrence par la production et rejette l'idée d'une médiation de la puissance publique locale, dans le cadre, par exemple, d'une procédure d'adjudication des concessions. Aussi les collectivités locales françaises ne peuvent-elles s'appuyer sur la Commission pour remettre en question la place centrale de l'État. Si ce dernier voit son mode d'intervention redéfini avec la création d'une nouvelle autorité de régulation - la Commission de Régulation de l'Électricité (CRE) -, avec le renforcement des pouvoirs de la Commission, il conserve toutefois certains instruments lui permettant d'orienter la mutation du secteur. Bien que non achevée à l'issue de la transposition de la directive européenne sur l'électricité19, l'ouverture du marché à la concurrence ne saurait donc, pour le moment, être apparentée à un processus de décentralisation, où les collectivités locales françaises joueraient un rôle de premier ordre (Poupeau, 2000).

Une autre lecture des changements actuels est cependant de mettre en évidence une "reterritorialisation" d'EDF. En effet, à la fin des années 1980, l'entreprise publique modifie son rapport aux collectivités locales. Un peu partout en France, des partenariats sont noués avec les villes, départements et régions dans des domaines aussi divers que le développement économique, la gestion des impayés ou la protection de l'environnement. Mais surtout, dans un contexte d'incertitude institutionnelle, EDF décide, pour la première fois depuis 1946, de reconsidérer ses liens contractuels avec les communes. Ce revirement peut a priori surprendre venant d'une entreprise qui a toujours considéré le pouvoir local comme source de déstabilisation potentielle pour son organisation verticale et intégrée. Deux raisons principales motivent une telle décision, qui nécessitent de considérer avec plus d'attention la stratégie d'EDF au tout début des années 1990.

19 la directive est transposée dans le droit français le 1er février 2000, avec un an de retard sur le calendrier fixé par les instances communautaires. En fait, seuls 30\% du marché français de l'électricité - les gros industriels - sont ouverts à la concurrence. 
A une période où l'entreprise publique se prépare à l'ouverture à la concurrence et se diversifie dans des activités de services urbains ${ }^{20}$, la contractualisation permet de renouveler en profondeur, à la fois en externe et en interne, une image de service public jacobine. S'accompagnant d'un gros effort de communication pour inciter les agents à s'ouvrir davantage sur leur environnement local, elle favorise de nouvelles pratiques de coopération et renforce l'ancrage institutionnel d'EDF.

La seconde raison, plus fondamentale, est d'ordre stratégique. Elle tient à la volonté des dirigeants d'EDF de combler le vide juridique existant depuis 1946 sur les contrats de concession, vide qui pourrait être mobilisé par les villes et les grands groupes de services urbains pour démanteler le système de distribution centralisé. Conçue d'emblée comme une parade contre une émancipation potentielle des villes, la contractualisation ne saurait conduire, pour EDF, à l'adoption d'un nouveau modèle de service public, qui serait local et non plus national. Dans ce domaine, les marges de manoeuvre de l'entreprise seraient d'ailleurs limitées par les prérogatives régaliennes en matière de tarification et d'investissement. Mais surtout, EDF n'a aucun intérêt à jouer la confrontation avec l'État, allié qui la protège contre une libéralisation trop rapide tout en lui permettant de se développer à l'étranger, et la CGT, qui brandit la menace d'un important mouvement social. L'ouverture d'un second "front" - la clientèle des particuliers par adjudication des concessions - risquerait, au contraire, de déstabiliser un peu plus une entreprise appelée à se battre pour conserver sa clientèle industrielle, objet de toutes les convoitises des concurrents. Aussi les dirigeants d'EDF préfèrent-ils jouer la carte du statu quo.

La stratégie de contractualisation reflète bien cette préoccupation de redistribution minimale des pouvoirs vers le local. Pour ce faire, les dirigeants d'EDF s'appuient sur la FNCCR, Fédération marquée par son implantation rurale - via les syndicats d'électrification - et qui défend le maintien de la centralisation et de la péréquation tarifaire ${ }^{21}$. Ensemble, les deux protagonistes négocient en 1991 un cahier des

\footnotetext{
${ }^{20}$ comme la vidéocommunication, le traitement des ordures ménagères ou la télésurveillance.

${ }^{21}$ qu'un retour au modèle de distribution d'avant-guerre aurait inévitablement remise en question. L'attachement d'une très large majorité d'hommes politiques et de Français à cette disposition, qui ne date que des années soixante, explique aussi, au-delà des stratégies propres à chacun des acteurs (EDF, FNCCR...), la stabilité du système de distribution électrique français.
} 
charges modèle, qui doit servir de base aux discussions locales. Les représentants des villes, membres d'associations d'élus ou de fonctionnaires territoriaux ${ }^{22}$, sont mis provisoirement à l'écart et informés tardivement, ce qui n'est pas sans susciter de vives protestations.

De la négociation avec la FNCCR sortent une série de dispositions qui visent à revigorer le pouvoir concédant et inciter les communes à signer le nouveau cahier des charges. Ces dispositions sont d'autant mieux acceptées par EDF et par les tutelles de l'État qu'elles ne représentent qu'un partage limité de la rente générée par l'activité de distribution. Première disposition majeure, EDF s'engage à participer à l'enfouissement des lignes de basse et moyenne tensions, suivant des modalités à définir localement entre centres de distribution et autorités concédantes. Au moment où de nombreux élus se préoccupent des questions d'esthétique des réseaux, cet engagement représente un levier d'action non négligeable. La seconde disposition, de loin la plus importante, est d'ordre financier. Deux redevances sont instaurées, l'une pour compenser les investissements consentis par les collectivités $\operatorname{locales}^{23}$, l'autre pour permettre à ces dernières d'assumer le contrôle de la concession. En contrepartie de son soutien, la FNCCR obtient que le montant de ces redevances soit fonction de la taille du pouvoir concédant, en nombre de communes et en population représentée. Certains groupements départementaux peuvent ainsi escompter toucher plusieurs dizaines de millions de francs. De la sorte, la FNCCR entend renforcer son assise locale en incitant les élus à créer de nouveaux syndicats ou à confier aux syndicats d'électrification existants le statut d'autorité concédante. A plus long terme, elle se voit être le représentant national d'un pouvoir concédant organisé par département, qui pourrait devenir un contrepoids face aux villes et aux futurs opérateurs.

Dès 1992, forts de cet outillage juridique et financier, les agents d'EDF, parfois aidés d'émissaires de la FNCCR, prennent leur bâton de pèlerin pour inciter les communes à se regrouper et adopter le nouveau cahier des charges. En 1992, déjà plus d'une dizaine de signatures sont enregistrées avec des syndicats de taille

22 notamment l'Association des Maires des Grandes Villes de France (AMGVF) et l'Association des Ingénieurs des Villes de France (AIVF).

23 car, de par la loi, les communes ont la possibilité d'investir elles-mêmes dans les réseaux électriques (ce qu'elles font d'ailleurs pour l'électrification rurale). 
départementale. Fin 1994, 56\% des communes françaises ont signé le nouveau cahier des charges, soit une cinquantaine de syndicats départementaux et 130 villes $^{24}$. Ce chiffre ne cesse de croître pour atteindre $84 \%$ des communes en 199725. Si les négociations ne posent pas de difficulté majeure avec la plupart des syndicats intercommunaux, elles sont plus ardues avec les villes, qui réclament davantage de contreparties financières et la possibilité de mieux coordonner les différents réseaux locaux d'énergie (électricité, gaz, réseaux de chaleur). Mais, face aux réticences manifestées par EDF, beaucoup d'entre elles préfèrent signer le cahier des charges, d'autant plus que les dispositions en faveur de l'environnement ou la signature de conventions annexes constituent des éléments attractifs.

Si le succès de l'intercommunalité peut différer d'une région à une autre, la tendance générale est à une bipolarisation des relations entre EDF et le monde local autour, d'une part, des villes, d'autre part, des départements. Bénéficiant d'une assise déjà ancienne, beaucoup de syndicats d'électrification rurale profitent de la contractualisation pour se doter d'une compétence d'autorité concédante. Ceux qui ont réussi à fédérer un grand nombre de communes voient leur budget s'envoler et peuvent s'affranchir de la tutelle technique de la Direction Départementale de l'Équipement (DDE) ou de la Direction Départementale de 1'Agriculture et de la Forêt ${ }^{26}$ (DDAF) en employant leur propre personnel. Dans certains syndicats, des équipes d'une dizaine de personnes sont formées pour gérer les dossiers. En ce qui concerne le contrôle d'EDF, ces équipes sont parfois épaulées par l'Association pour l'Expertise des Concessions (AEC), créée par la FNCCR pour donner une plus forte consistance au pouvoir concédant. En réalisant ses propres enquêtes sur la satisfaction des clients, en auditant les comptes des centres, l'AEC contribue à diffuser une expertise locale en matière de distribution électrique. On ne saurait donc comparer les syndicats départementaux aux structures d'électrification rurale qui existaient précédemment et qui, dans la majorité des cas, se cantonnaient à une fonction de courroie de transmission des crédits du FACE vers les syndicats primaires. Désormais, une " technostructure " tend à se constituer, qui dispose de moyens financiers et humains accrus.

\footnotetext{
${ }^{24}$ source : FNCCR (congrès 1994).

${ }^{25}$ source : FNCCR (congrès 1997).

${ }^{26}$ qui ont succédé dans les années 1960 respectivement aux Ponts-et-Chaussées et au Génie Rural.
} 


\subsection{Redéfinir l'ancrage territorial d'EDF : la mise en oeuvre d'une démarche de contractualisation interne}

De manière parallèle à ce processus de " refondation ", EDF engage une politique de redéploiement territorial. Dès 1946, la question de la rationalisation des structures de la distribution ne cesse d'alimenter les réflexions au sein de l'entreprise. Des rapports internes se succèdent pour rechercher des économies d'échelle dans une Direction où le coût de la main d'oeuvre est par nature très élevé. Ils ouvrent la voie à des débats récurrents entre les partisans d'une suppression des Directions Régionales et ceux qui sont hostiles au maintien des subdivisions ${ }^{27}$. Cependant, face aux réticences syndicales, aucune action de grande envergure n'est amorcée. La rationalisation se limite à des suppressions ponctuelles d'unités, à des expérimentations dans quelques Directions Régionales et à l'introduction de l'informatique ou d'instruments de gestion pour gagner en productivité.

La question revient avec force dans les années 1980 avec la signature du contrat de plan État-EDF puis avec la perspective de l'ouverture du marché français à la concurrence. Pour les dirigeants d'EDF, la compétitivité de l'entreprise passe par une révision radicale de son implantation ${ }^{28}$. Aussi, parallèlement à une mutation culturelle et managériale ${ }^{29}$, la Direction de la Distribution entame-t-elle, dès 1990, une refonte de ses structures. Celle-ci repose sur un choix institutionnel fort, en congruence avec le mouvement de négociation des contrats de concession : le renforcement du centre et, incidemment, du système départemental. La région, qui aurait pu être privilégiée, n'est pas retenue. Échelon jugé éloigné des réalités locales mais surtout perçu comme une menace pour l'entreprise ${ }^{30}$, les Directions

27 en fait, subdivisions et districts ont changé de nom dans les années quatre-vingt. Les premiers sont devenus "agences", les seconds ayant été souvent scindés en "agences d'exploitation" et "agences clientèle". Il existe encore quelques districts au milieu des années quatre-vingt-dix. Par souci de clarté, nous continuerons à employer les anciens termes

28 au début des années 1990, on estime à EDF que, pour réduire de $5 \%$ en cinq ans le prix de vente du kWh, objectif assigné par le contrat de plan État-EDF, il faut réduire de $20 \%$ les frais de personnel (Ménard et Barreau, 1997).

29 l'introduction de la notion de service bouleverse l'ancien modèle bureaucratique. Dorénavant, le client est placé au coeur de l'organisation, ce qui se traduit notamment par la création d'agences (résidentiel, tertiaire, entreprises, voire collectivités locales), qui regroupent des interlocuteurs autrefois dispersés (commerciaux, clientèle, techniciens). Pour de plus amples développements, cf Berrivin, 1995 ; Poupeau, 1999

30 à la fois sur le plan organisationnel (les Directions Régionales sont devenues, au fil des années, de véritables écrans entre les services parisiens d'EDF-GDF et les centres de distribution) et 
Régionales sont supprimées au profit d'une coordination inter-centres plus lâche. Sur le plan managérial, le directeur de centre acquiert davantage de latitude pour remettre en cause le poids des filières techniques et des chefs de subdivision et de district. Il a pour mission de repenser l'insertion de son unité dans l'environnement local, notamment en valorisant les domaines commercial et clientèle. Pour ce faire, il est chargé de définir un "Plan Stratégique de Centre", dans lequel doivent apparaître, à partir d'un diagnostic local, les principales orientations qu'il compte suivre.

Dans le nouveau schéma, subdivisions et districts doivent être, à terme, supprimés. Pour les remplacer, les responsables de la Direction EDF-GDF Services $^{31}$ (DEGS) préconisent une démarche de contractualisation interne, qui s'inspire des théories micro-économiques sur les contrats ${ }^{32}$. D'une taille variable de trois à plus d'une quinzaine de personnes -, des Groupes Responsables (GR) sont constitués dans les centres. Ils dépendent d'un Comité de Direction, organe rassemblant tous les chefs de services et placé sous la responsabilité du directeur. Les GR correspondent à un type de métier particulier : l'exploitation des réseaux, le développement des ventes, la gestion de la clientèle... Afin de mieux délimiter les domaines d'action, chaque GR est doté de compétences précises et négocie un budget annuel avec le Comité de Direction. L'efficacité du groupe est appréciée en fonction d'objectifs définis en début d'année, suivant une série de critères essentiellement quantitatifs. Ainsi, un GR “accueil clientèle" sera évalué au regard du nombre de clients " traités" par agent, du temps d'attente téléphonique, du taux de satisfaction des clients... A terme, de verticale et soumise aux logiques de filières, l'organisation doit muer vers un mode de coordination plus horizontal, centré sur les métiers et étroitement piloté par le directeur de centre.

Lancée en très peu de temps en 1990, la réforme met plusieurs années à se mettre en oeuvre. Peu de centres passent directement du modèle pyramidal au modèle contractuel. En fonction du climat social et des spécificités locales, certaines unités commencent par supprimer les subdivisions, d'autres les districts. Cette

institutionnel (les dirigeants d'EDF craignent qu'en suivant l'exemple de l'Angleterre, certains réformateurs ne soient tentés de démanteler la Distribution en établissements publics régionaux).

31 il s'agit du nouveau nom donné à la Direction de la Distribution d'EDF-GDF.

32 Berrivin, 1995. Sur l'utilisation du "contrat" dans le management public, cf. également Sociologie du Travail, 1996. 
stratégie de changement progressif suscite des tensions et des difficultés d'appropriation car l'organisation en GR doit souvent cohabiter, dans les premiers temps, avec l'ancienne structure pyramidale. Pourtant, le nouveau schéma finit par s'imposer, comme en atteste l'état actuel des réformes dans les centres.

Cette nouvelle organisation n'est pas sans conséquences sur la nature des relations que les unités de la DEGS entretiennent avec leur environnement local. Parmi les acquis considérés comme positifs par les élus et les fonctionnaires territoriaux, une plus grande ouverture est à mettre au crédit de la réforme. Elle participe du mouvement de " reterritorialisation" engagé par EDF sur le plan institutionnel. La création d' "agences collectivités locales", d'interlocuteurs privilégiés des communes, de GR spécialisés clarifie les relations et valorise l'écoute des revendications des élus. Par exemple, pour ce qui concerne les contrats, toutes les factures sont désormais traitées par un seul individu, alors qu'auparavant, elles l'étaient à la fois par les centres, les subdivisions et les districts, suivant la nature des tarifs. Les collectivités locales ont affaire à un interlocuteur unique, qui peut intervenir plus rapidement en cas de problème car il maîtrise l'ensemble des contrats. En outre, cet agent assure un suivi des factures, propose de nouveaux services, repère les surconsommations et se déplace dans les communes pour réaliser un bilan annuel, ce qui ne se faisait pas auparavant ${ }^{33}$.

Pour positive qu'elle soit vécue sur ce plan, la réforme de la Distribution fait cependant apparaître quelques mécontentements, qui sont symptomatiques des nouvelles formes de régulation qui s'instaurent. Ils sont, pour l'essentiel, formulés par les représentants des communes rurales, individuellement ${ }^{34}$ ou par le biais de leurs canaux représentatifs nationaux ${ }^{35}$. Ceux-ci dénoncent, en premier lieu, ce qu'ils estiment être un retrait d'EDF des territoires et une menace pour le maintien du service public dans les campagnes. Mais cette insatisfaction traduit aussi une perte d'influence au quotidien. Avant qu'ils ne disparaissent ou soient concurrencés par les GR, les chefs de subdivisions et de districts étaient des acteurs-clés des relations avec les élus. Disposant de marges de manoeuvre budgétaires, pouvant mobiliser des ressources variées, ils étaient en mesure de

\footnotetext{
33 ou alors, c'était sous la pression d'un élu local, qui obtenait ce service à titre dérogatoire. Le changement provient du caractère systématique de la démarche.

34 à en croire les enquêtes de satisfaction réalisées par la DEGS.

35 notamment la Fédération Nationale des Maires Ruraux (FNMR).
} 
répondre à certaines sollicitations ${ }^{36}$. Avec les réformes, les demandes transitent davantage par les centres. Soumis à un contrôle budgétaire plus strict, disposant d'un domaine de compétence circonscrit, souffrant d'une tendance générale des agents à se focaliser sur leurs propres objectifs (Berrivin, 1995), les chefs de GR éprouvent parfois plus de difficultés à satisfaire les demandes. Les arbitrages remontent de plus en plus vers le Comité de Direction du centre, qui supervise les GR et tente d'instaurer une coopération plus transversale.

D'une manière générale et malgré ses nombreuses limites (Berrivin, 1995), la réforme de la Distribution a permis aux directeurs de renforcer l'emprise de l'échelon centre. Le renforcement est manifeste dans les unités ayant généralisé la contractualisation interne. Mais, même dans celles où les GR cohabitent avec l'ancienne structure, le nouveau modèle de régulation tend à s'imposer. Disposant de pouvoirs renforcés, d'une légitimité accrue, les directeurs ne sont plus, à l'image des anciens chefs de centre, des personnages dont l'autorité était contestée par des chefs de services puissants, des syndicats mobilisateurs et des chefs de subdivisions et de districts fortement implantés. Beaucoup ont su affirmer leur leadership et affaiblir la logique de filière qui caractérisait le fonctionnement passé. Au moment où le processus de libéralisation tend à s'accélérer, le centre apparaît donc comme l'échelon ayant le plus bénéficié des changements.

\subsection{Le système départemental au coeur du redéploiement d'EDF}

De ces évolutions résulte une profonde recomposition du "modèle EDF". La pierre angulaire en est le système départemental, c'est-à-dire à la fois l'échelon centre de la DEGS et les syndicats d'électrification devenus autorités concédantes de la distribution d'électricité. Ces derniers s'imposent comme des interlocuteurs privilégiés pour les centres, non seulement pour le contrôle de la concession mais aussi pour l'enfouissement des lignes. Ils se positionnent de plus en plus comme les médiateurs des demandes des communes rurales. Si les coopérations sont

\footnotetext{
${ }^{36}$ par exemple, affecter durant une journée des agents dont ce n'était pas la fonction à la relève de tous les compteurs d'une commune parce qu'un élu exigeait d'avoir une seule période de facturation (mobilisation de ressources en personnel) ou prendre des crédits sur le budget travaux - et donc retarder d'autres opérations - pour répondre à la demande impromptue d'un maire qui souhaitait coordonner les chantiers sur un tronçon de voirie (mobilisation de ressources financières).
} 
variables avec les Conseils Généraux ${ }^{37}$, ces syndicats contribuent à conforter l'échelon départemental sur la scène institutionnelle locale.

Dans ce contexte, seules les communes importantes ont su préserver une certaine capacité d'action, d'une part parce qu'elles ont conservé leurs prérogatives d'autorités concédantes, d'autre part parce qu'elles représentent un axe de développement important pour EDF. La multiplication des partenariats montre la volonté de l'entreprise de ménager ces acteurs, dont le rôle ne saurait que s'accroître avec la libéralisation du marché de l'énergie. En revanche, comme nous l'avons souligné, les communes rurales ont peine à s'affirmer. Souffrant de la rationalisation de l'activité de distribution, elles apparaissent aujourd'hui plus périphériques dans la régulation quotidienne du service public de l'électricité.

\section{CONCLUSION}

Le redéploiement d'EDF met donc en exergue le dynamisme du système départemental français, qui a su tirer profit des évolutions récentes pour renforcer son poids sur la scène locale. La vitalité de cet échelon, souvent critiqué pour ce qui serait son immobilisme ou son caractère désuet, peut a priori surprendre dans le contexte de mondialisation du secteur énergétique, qui s'accompagne, dans certains pays étrangers, d'une montée en puissance des villes et des régions. Tel n'est cependant pas le cas en France, où le département continue toujours, pour le moment, d'être le point de référence institutionnel local et ce malgré l'amorce récente par EDF d'une régionalisation de son activité de distribution ${ }^{38}$.

Cette particularité tient en grande partie à la volonté des dirigeants d'EDF de ne pas sortir du modèle de service public issu de la nationalisation mais, au contraire, de s'appuyer sur le monde rural pour consolider les bases de leur entreprise, au moment où se dessine la libéralisation du marché français. Il faut donc accorder une importance toute particulière à EDF et à sa capacité à façonner le futur

\footnotetext{
37 les syndicats sont souvent dirigés par des "seconds couteaux" politiques des départements, sénateurs et/ou conseillers généraux. Pour ces élus, la tentation est grande d'utiliser ces structures intercommunales pour participer à des opérations pilotées par le Conseil Général.

38 il serait plus exact de parler de mutualisation de certaines ressources à l'échelon régional car ce mouvement ne s'accompagne pas de nouvelles structures, mises à part les Agences Commerciales qui ont été créées en 1998 pour les marchés de la grande industrie et du gros tertiaire.
} 
paysage énergétique. Toutefois, en négociant les termes de son soutien, par l'entremise de la FNCCR, le monde rural départemental n'a pas joué un rôle passif. Sachant mobiliser des ressources à la fois juridiques, politiques et financières, il a réussi à se repositionner sur la scène institutionnelle locale. Il apparaît désormais comme un partenaire avec lequel l'ensemble des opérateurs devront compter.

Ce dynamisme départemental n'est pas propre à l'électricité mais se manifeste aussi dans d'autres services publics industriels et commerciaux. Des syndicats départementaux s'investissent dans le câble, les réseaux de télécommunications ou les nouvelles technologies de l'information pour pallier ce qu'ils estiment être des déficiences des opérateurs privés. D'autres syndicats se constituent pour encadrer la distribution de gaz, qui, à l'instar de l'électricité, est soumise à un régime de service public local. Regroupant parfois de vastes ensembles intercommunaux, ils négocient avec Gaz de France et les pouvoirs publics de meilleures conditions de desserte pour les zones rurales qui, à la différence de l'électricité, ne sont pas toutes irriguées. La collecte et la valorisation des ordures ménagères connaissent, elles aussi, un large mouvement de départementalisation, qui structure fortement l'organisation de la filière du traitement des déchets. Enfin, dans deux secteurs traditionnellement considérés comme le "fief" des grands groupes de services urbains, l'eau et d'assainissement, des voix se font entendre pour favoriser l'émergence de syndicats départementaux, qui pourraient contrebalancer le poids des villes et des fontainiers.

Ce foisonnement d'initiatives, s'il en est encore à ses prémisses, soulève une question d'ordre plus théorique, qui a trait à la transformation du mode de gouvernement départemental. Après avoir été longtemps au coeur des analyses portant sur le système politico-administratif local, le département n'a pas connu l'engouement des chercheurs pour les agglomérations urbaines. Au contraire, coincé entre des villes en plein développement et des régions qui occupent une place croissante dans l'organisation territoriale française, il fait figure, pour certains, de vestige de la France d'avant-guerre. Pourtant, face aux critiques dont il est l'objet, le système départemental réagit. En redéployant de vieilles structures héritées de la Troisième République - comme le sont les syndicats d'électrification rurale -, il accompagne le mouvement général de redéfinition du rôle de l'État dans l'économie. Il se positionne dans la réorganisation du service public. Il tente de 
jouer un rôle dans l'approfondissement de l'intercommunalité, qu'elle soit " de gestion" ou "de projet 39 ". A l'instar des villes, même si elles s'opèrent apparemment à une plus petite échelle, ces évolutions mettent en évidence le brouillage qui existe entre acteurs privés et publics, entre niveaux d'administration territoriale, autant d'éléments tendant à rendre plus complexe l'analyse du système local. Ces évolutions, dont les contours sont encore au stade de l'ébauche, nous invitent à affiner nos concepts et porter un regard plus attentif à la transformation de l'action et du mode de gouvernement départemental.

\section{BIBLIOGRAPHIE}

Baumol, W. J., Panzar, J. C., Willig, R. D., 1982. Contestable Markets and the Theory of Industry Structure. Harcourt Brace Jovanovich, New York.

Berrivin, R., 1995. La contractualisation des relations centre-périphérie au ministère de l'Équipement et à EDF. Thèse de l'Institut d'Études Politiques de Paris.

Bonaïti, J.-P., 1997. La décentralisation dans la loi de 1946. Du compromis introuvable à l'accommodement implicite. In : Association pour l'histoire de l'électricité en France (Ed.), La nationalisation de l'électricité en France. Nécessité technique ou logique politique? Paris, Association pour l'histoire de l'électricité en France, pp. 173-193.

Crozier, M., Thoenig, J.-C., 1975. La régulation des systèmes organisés complexes, Revue Française de Sociologie 16 (1), 3-32.

Crozier, M., Friedberg, E., 1977. L'acteur et le système. Le Seuil, Paris.

Curien, N. (dir.), 1992. Économie et Management des entreprises de réseau. Économie des réseaux, réseaux organisateurs et management en réseau. Economica, Paris.

Flux, 1998. Numéro spécial (31-32).

\footnotetext{
39 les syndicats d'électrification, d'eau, d'assainissement (...) font partie de la première catégorie, la plus ancienne, par opposition aux communautés de communes, aux communautés d'agglomération et aux communautés urbaines, qui appartiennent à une intercommunalité dite " de projet".
} 
Grémion, P., 1976. Le pouvoir périphérique. Bureaucrates et notables dans le système politique français. Le Seuil, Paris.

Henry, C., 1997. Concurrence et services publics dans l'Union européenne. PUF, Paris.

Jouve, B., Lefèvre, C., 1999. De la gouvernance urbaine au gouvernement des villes? Permanence ou recomposition des cadres de l'action publique en Europe ?, Revue Française de Science Politique 49 (6), 835-853.

Le Galès, P., 1995. Du gouvernement des villes à la gouvernance urbaine, Revue Française de Science Politique 45 (1), 57-95.

Lorrain, D., 1991. De l'administration républicaine au gouvernement urbain, Sociologie du Travail 33 (4), 461-484.

Lorrain, D., 1993. Après la décentralisation, l'action publique flexible, Sociologie du Travail 35 (3), 285-307.

Lorrain, D., 1998. Administrer, gouverner, réguler, Les Annales de la Recherche Urbaine (80-81), 85-92. 
Ménard, J.-Y., Barreau, J., 1997. Stratégies de modernisation et réactions du personnel. Le cas de trois entreprises publiques d'électricité : Électricité De France, Hydro-Québec \& Société Hongroise d'Électricité. L'Harmattan, Paris.

Morsel, H., 1996. Histoire de l'électricité en France. Fayard, Paris (tome 3 : 19461987).

Poupeau, F.-M., 1999. EDF ou la permanence d'un " compromis républicain ". Le système de distribution électrique français entre État et collectivités locales, de la nationalisation à la mondialisation. Thèse de l'Institut d'Études Politiques de Paris.

Poupeau, F.-M., 2000. Un néo-libéralisme centralisateur. Les collectivités locales dans la libéralisation du système de distribution électrique français, Politiques et Management Public 18 ( 2), 1-24

Sociologie du Travail, 1996. Contrats et pratiques contractuelles. Approches pluridisciplinaires, numéro spécial 38 (4).

Veltz, P., 1996. Mondialisation, villes et territoires. L'économie d'archipel. PUF, Paris.

Wieviorka, M., Trinh, S., 1989. Le modèle EDF. La Découverte, Paris. 